\title{
What is new in our understanding of pressure injuries: the inextricable association between sustained tissue deformations and pain and the role of the support surface
}

\section{Gefen A and Soppi E}

Keywords pressure ulcer, inflammation, ischaemia, support surface, coronavirus disease 2019

For referencing Gefen A \& Soppi E. What is new in our understanding of pressure injuries: the inextricable association between sustained tissue deformations and pain and the role of the support surface. Wound Practice and Research 2020; 28(2):58-65.

DOI https://doi.org/10.33235/wpr.28.2.58-65

\section{Abstract}

This paper first provides an overview of the contemporary research findings and latest aetiological discoveries concerning the prevention of pressure injuries (PIs) as reported in the 2019 version of the Prevention and treatment of pressure ulcers/injuries: clinical practice guideline (known as the International guideline), including, where relevant, through the new prism of the coronavirus disease 2019 (COVID-19) pandemic. Second, the biomechanical principles of PI prevention (PIP) through minimisation of tissue deformation levels are explained from a support surface design perspective. Third, and related to the second, the association between alleviation of sustained tissue deformations and mitigation of PI-risk-related or PI-related pain are reviewed with a focus on the role of the support surface. Fourth, and last, a discussion of the current $\mathrm{PI}$ aetiology theory is presented from a clinical practical perspective, using one documented patient testimony and two additional patient case stories, which are used here

\section{Amit Gefen* PhD}

Professor of Biomedical Engineering and

the Herbert J. Berman Chair in Vascular

Bioengineering, Department of Biomedical

Engineering, Faculty of Engineering,

Tel Aviv University, Tel Aviv, Israel

Email gefen@tauex.tau.ac.il

Esa Soppi MD

Eira Hospital, Helsinki, Finland

* Corresponding author to analyse the complex interlinks between the known aetiological factors in Pls - discomfort and pain.

\section{Introduction}

Pressure injuries (PIs) make a considerable portion of all chronic wounds and are one of the most important, unsolved and costliest medical problems. The problem of Pls escalated remarkably since February-March 2020 when the coronavirus disease 2019 (COVID-19) outbreak inflated the global numbers of patients who require admission to an intensive care unit (ICU) and who are anaesthetised and mechanically ventilated there. Such patients, either ventilated supine (including those with respiratory insufficiency necessitating extracorporeal membrane oxygenation (ECMO) treatment) or positioned prone (typically to improve tissue oxygenation in acute respiratory distress syndrome (ARDS) condition) are constantly susceptible to Pls. Their hospital-acquired PIs may be caused by both bodyweight forces and the intensive use of medical devices on their skin surface, for example ventilation masks, endotracheal and nasogastric tubing, catheters, electrodes, oxygen saturation and temperature probes $^{1,2}$.

Already prior to this extreme additional burden of the COVID19 pandemic, clinical protocols for PI prevention (PIP) are not straightforward to implement and, even if implemented successfully, they certainly do not guarantee zero PI incidence rates. A primary reason for this is that the risk for a PI highly depends on the individual anatomical characteristics and physiological system function, as well as on any other injury or disease, either at an acute or a chronic stage ${ }^{3,4}$. Our bioengineering research work published in the last 2 decades has explained why absolute and generic injury thresholds 
to predict when Pls may occur will forever remain elusive, despite the massive efforts and resources that have been invested in allegedly discovering universal injury thresholds to predict when a PI will occur ${ }^{3,4}$. The individual susceptibility to Pls will always depend on integrated body system functions which are dynamic and extremely difficult to predict in seriously ill patients. Yet, there is new understanding and promising research routes for contemporary work which already leads to better PIP and treatment of existing PIs even if, currently, it is not feasible to predict when a specific person may be affected. This new knowledge is reviewed here, with some updates through the COVID-19 prism, with a specific focus on the complex relations between PI aetiological factors as described in the 2019 Prevention and treatment of pressure ulcers/injuries: clinical practice guideline ${ }^{5}$ (known as the International guideline) - discomfort and pain. The importance of selection of an adequate support surface to relieve the sustained tissue deformations, discomfort and pain is also highlighted in the above context.

\section{Findings}

\section{Understanding the aetiology of pressure injuries}

Pressure injuries develop over a timescale of minutes to hours under sustained tissue deformations ${ }^{6}$. Tissue damage in Pls does not appear instantaneously, but rather develops from the cell scale to the mesoscale and tissue level and, finally, presents itself on the skin surface, often causing skin and underlying tissue breakdown. This implies that a damage spiral onsets and progresses from micro to macro. Our current fundamental understanding described in the 2019 International guideline is that this damage spiral ultimately leading to Pls is triggered and then driven by cell and tissue exposure to sustained mechanical deformations or, in bioengineering terms, to mechanical stress concentrations in soft tissues (Figure 1).

Any bodyweight or device-related forces which cause sustained soft tissue distortions generate large deformations of the cells contained within the affected tissues, with the greatest tissue and cell deformations occurring where these forces are concentrated (Figure 1). With respect to sustained bodyweight forces, the most influenced soft tissue sites are typically found under bony prominences, where the highly curved and 'sharp' bone surfaces come into contact with easily deformable muscle, adipose or skin tissues. The bodyweight forces which are transferred through the sharp and rigid bony elements cause large distortions in the soft tissue structures that they encounter (Figure 1). A similar scenario would apply for any external device, for example a continuous positive airway pressure (CPAP) mask or oxygen saturation probe which deliver forces onto the skin of a patient. At the cell scale, the continuous exposure to such mechanical forces that deform soft tissues would gradually damage the integrity of the cytoskeleton - the complex protein scaffold which makes the structural framework of cells. The exterior cell walls, called the plasma membrane, are structurally supported by the cytoskeleton. When the cytoskeleton becomes unable to continue providing the sufficient mechanical support to the plasma membrane, pores will form on the membrane ${ }^{7}$. Poration of the plasma membrane will rapidly lead to abnormal transport of ions and molecules from within cell bodies extracellularly, and from the extracellular space inwards into the cell bodies. The inability of multiple cells to control their mass transport yields collective loss of homeostasis which results in apoptotic cell death within a timeframe of just minutes ${ }^{1-5}$.

Figure 1. Deformations of soft tissues and cells across the different dimensional scales. When a patient is positioned on a support surface, for example in a supine posture, their bony prominences including the sacrum are compressing and deforming the soft tissues contained between the bony region and the support surface. For example, the rigid and sharp sacral structure (shown in a low-dose computed tomography scan at the centre frame) creates a mechanical stress concentration in soft tissues in its vicinity, which is best visualised using computer modelling. Cells within this stress concentration zone will be highly distorted and deformed (lowest right-hand side frame), whereas cells that are relatively distant from the stress concentration zone (lowest left frame) will be less distorted. The nearer the cells are to the stiff and curved sacrum, the greater the stress level is. Cells located in deep soft tissues directly under the sacrum of a supine patient and in its vicinity will therefore be the first to be affected by deformationinduced cell damage, which may ultimately manifest as a clinical deep tissue injury that may further progress to a full-thickness PI.

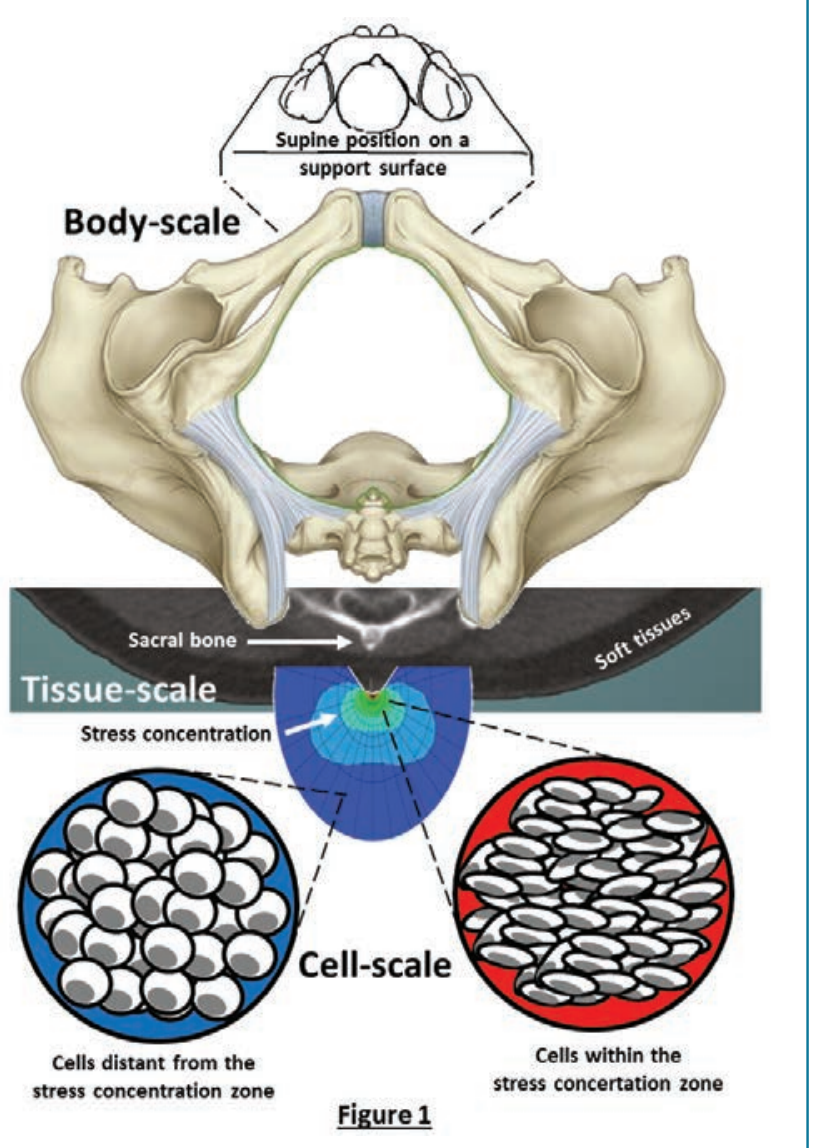


When multiple cells have been damaged or have died as a direct result of the sustained tissue deformations as described above, the damaged cells and nearby immune cells release pro-inflammatory cytokines which are signalling proteins that function to attract additional immune cells. Examples for such pro-inflammatory cytokines which become over-expressed in the inflammatory phase of a PI are interleukin-1 $\alpha$ (IL-1 $1 \alpha)$ and tumour necrosis factor- $\alpha(\text { TNF- } \alpha)^{8}$. This signalling is a programmed normal response which is essential for healing. Recruitment of a large number of immune cells is primarily aimed at counteracting pathogens, clearing dead cell debris, and preparing the ground for tissue regeneration. However, in the specific context of PI aetiology, the inflammatory singling itself is a potential contributor to the injury spiral, considering the effects of the pro-inflammatory cytokines on the endothelium in the vasculature adjacent to the initial damage site. The secreted pro-inflammatory cytokines act to dilate capillaries and increase the permeability of capillary walls near the initial damage site by relaxing endothelial cell tight-junctions. This endothelium relaxation facilitates leukocyte extravasation - the migration of immune cells from the blood circulation to the initial damage site.

Figure 2. The inextricable and strong coupling between the vicious cycle of $P I$ formation and the discomfort and pain associated with a forming PI or an early-stage (microscopic) tissue damage preceding a 'clinical' Pl. Pathologic pain may originate from inflammatory, nociceptive or ischaemic sources, or a combination of these. All the above pain factors escalate and intensify as the PI progresses.

Sustained cell and tissue deformations are always the triggering event and the driving cause for the PI vicious cycle. Accordingly, alleviating the exposure of an at-risk patient to sustained localised tissue deformations should be highly effective in reducing an associated discomfort or pain sensation.

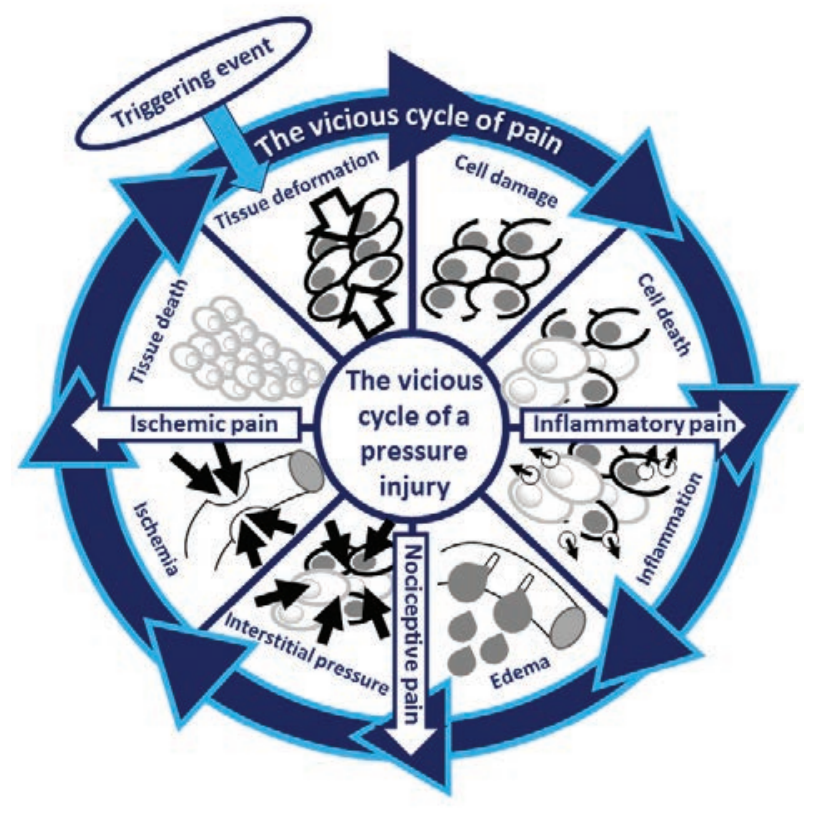

However, the endothelium relaxation also results in leakiness of the vasculature near the damage site and so, plasma fluids build up in the interstitial tissue spaces, which forms oedema. Often, in a developing $\mathrm{PI}$, soft tissue expansion due to a forming oedema is mechanically limited, for example because the tissues are constrained between a bony element and a support surface (for example between the sacrum and a mattress), or between a bone surface and a nearly-rigid device (for example the facial tissues between the skull bones and a CPAP mask). If such soft tissues cannot sufficiently expand in volume, the interstitial pressures would increase sharply, causing further cell deformation and, thereby, additional deformation-induced cell death.

Under such conditions, the inflammatory process would then cause release of reactive oxygen and nitrogen species to degrade the extracellular matrix (ECM) in an effort to relieve the rising interstitial pressures, which will cause further tissue damage, now to the ECM. At a certain stage, the growing interstitial pressures may reach a level that would cause obstruction of the vasculature itself which will impair blood perfusion into the affected tissue site and thereby trigger ischaemic damage. These synergistic interactions between sustained cell and tissue deformations, inflammation and ischaemia form the vicious cycle of the development and progression of Pls as we currently understand it (Figure 2) and are always specific to the individual patient, depending on their respective body system functions ${ }^{9}$.

In the context of the current spread of COVID-19 where many of the newly admitted ICU patients who are anaesthetised for mechanical ventilation are, by definition, at risk for Pls, it is interesting to discuss how COVID-19 interacts with the known aetiological factors described above (Figure 2). First, COVID-19 activates the immune system promptly and sharply ${ }^{10}$ which positions COVID-19 patients with a cytokine release syndrome (CRS, also known as cytokine storm) at a high risk for developing PI-related inflammatory tissue damage. This is because their inflammatory response is unleashed and their cytokine sensitivity thresholds are therefore disrupted.

In addition, COVID-19 patients are also at a high risk for PI-related ischaemic tissue damage as their oxygen saturation levels are typically low and their cardiac output may be abnormal - for example due to myocarditis, acute myocardial infarction or heart failure - all of which have been documented as possible cardiovascular complications of COVID-1911. Another potential contributor to tissue ischaemia in COVID-19 is the hypercoagulability leading to a tendency for thrombosis (or micro-thrombi, also known as thrombotic showers) in these patients ${ }^{12}$. Some common COVID-19 treatments given at the ICU setting may also exacerbate the above key contributors to Pls, for example the use of vasopressors and inotropes that influence the vascular and immune systems as a secondary contributor ${ }^{13}$. 
These timely examples illustrate how COVID-19 interacts directly with two of the three primary aetiological factors in Pls - inflammation and ischaemia (Figure 2) - and suggest that COVID-19 may be a confounder of Pls. Indeed, anecdotal clinical data collected by collaborators of the authors over the last month suggest that the prevalence rate of hospital-acquired PIs in ICUs among COVID-19 patients could be 10-times or more the respective ICU PI rates at the same ICUs prior to the COVID-19 outbreak. Considering that Pls are also a well-recognised independent prognosticator of death among (non-COVID-19, general) ICU patients ${ }^{14}$, the interaction of the CRS in COVID-19 patients with the inflammatory damage factor in the PI spiral (Figure 2) underpins the importance of PIP for this particular patient population, particularly including an adequate selection of a support surface, as reviewed below.

To summarise this part, we now understand that the three key contributors to Pls - namely, direct deformation damage which is the primary factor, then the inflammatory damage which is secondary, and the ischaemic damage which is tertiary - degrade and exacerbate the state of cells and tissues altogether-5. Each of these three key factors is activated chronologically, at a different time point and distinct rate of contribution to the cumulative damage, depending on the individual characteristics, for example their anatomy and tissue composition and their inflammatory and cardiovascular system functions. Nevertheless, the exposure to sustained cell and tissue deformations is always the triggering event which commences and drives the damage spiral (Figure 2). This applies to both bodyweight-force-related Pls and to device-related Pls. Accordingly, the single most crucial and effective action in protecting patients from either type of Pls should be to minimise their exposure to sustained tissue deformations to the best possible extent.

Support surface design in pressure injury prevention

Our published bioengineering research demonstrated, experimentally and through computer modelling, that designing a support surface which provides adequate immersion and envelopment is the most effective approach for minimising the exposure of at-risk patients to sustained tissue deformations, which protects them from $\mathrm{Pls}^{15}$. Immersion is the depth into which the body of a patient penetrates when on a support surface, whereas envelopment is the intimacy in the contact of the body with the support surface.

The International Organization for Standardization (ISO) defines the characteristic of envelopment as the ability of a support surface to conform, so to fit or mould around the irregular shape of the human body. Some immersion is always required for achieving envelopment, that is, without immersion there is negligible envelopment. A good envelopment implies that local areas of interface pressure and shear are reduced by effectively supporting more tissues at the surface of the body ${ }^{15,16}$. In other words, as the contact area between the support surface and the body contours increases through envelopment, bodyweight loads are transferred more uniformly, minimising the potentially perilous areas of elevated tissue deformations, as explained above. The larger the contact area for the bodyweight load transfer is, the lesser the localised cell and tissue deformations are. As the exposure to sustained cell and tissue deformations is the triggering event and primary driver of the $\mathrm{PI}$ vicious cycle (Figure 2), a support surface that continuously provides good envelopment, regardless of the patient characteristics and body habitus, and which is also able to do so for all possible body positions, will be effective in PIP.

Other important features affecting the sustained tissue loading state of the user are the coefficient of friction (COF) of the skin-facing layer of the support surface (for example an overlie, bedsheet or transfer sheet) and the thermal properties of the support surface. The frictional forces acting on skin are proportional to the COF of skin with the skin-interfacing layer of the support surface. The thermal properties of the support surface also play a role in this regard - elevated skin temperatures leading to increased localised perspiration, build up of moisture and, thereby, a greater skin COF (mostly due to adhesive friction) cause higher frictional forces on skin and greater sustained shearing in underlying soft tissues ${ }^{17,18}$.

Sustained cell and tissue deformations, discomfort and pain

Pain has both physical and emotional constituents ${ }^{19}$. Physical noxious stimuli, including mechanical stimuli due to sustained tissue deformations, activate peripheral sensory neurons called nociceptors which, in turn, transmit signals to the spinal and supra-spinal nuclei, and from there to the medulla oblongata in the brainstem. There are a number of mechanisms at play in detecting and responding to pain, namely:

- The ability to detect pain via a nociceptive response (as reviewed below). Anaesthetic and sedative drugs, for example diminish the natural nociceptive response and thereby increase the PI risk.

- The ability to adequately process the 'pain message' (delivered through the neural system in a form of electrical impulses) within the central nervous system (CNS); this ability may be compromised in some patients, for example due to impaired brain activity.

- The capacity to respond to the above pain message, for example under conditions of a neuromuscular damage or disease.

Focusing now on the nociceptive response, nociceptors exist in skin, adipose, ligament, tendon and other connective tissues as well as in skeletal muscle tissues, that is, practically in all tissues that are susceptible to Pls. Nociceptors respond to different types of potentially noxious stimuli - including mechanical, thermal and chemical stimuli - and mediate deep tissue discomfort and pain ${ }^{20}$. The ability to detect such potentially tissue-damaging stimuli and respond to 
them timely, through postural changes (including micromovements), is distinguishing healthy persons who are protected from Pls from those individuals with impaired sensation. The latter group are prone to tissue damage because they lack a functioning nociceptive mechanism to detect and respond to deep tissue discomfort and pain. The susceptibility to deformation-inflicted tissue damage (Figure 2) can be due to peripheral neural damage such as in neuropathies, or due to a high-level nerve injury as in femoral and sciatic nerve blocks ${ }^{21}$, spinal cord injuries, stroke or brain trauma.

\section{Current pressure injury aetiology theory}

Importantly, with regards to the contemporary understanding of $\mathrm{PI}$ aetiology depicted in Figure 2, inflammation and discomfort/pain are strongly linked together through a complex signalling process which is a fundamental part of the healing physiology ${ }^{5}$. Specifically, pro-inflammatory cytokines contribute not only to the onset and maintenance of inflammation but also to the development of discomfort and pain by stimulating nociceptors ${ }^{22}$. The specific proinflammatory cytokines interleukin-6 (IL-6) and TNF- $\alpha$ are both known to play important roles in pathologic pain ${ }^{23,24}$. In addition, inflammation is considered to lower the threshold for pain perception in the CNS, leading to hyperalgesia ${ }^{25}$. Lastly, elevated concentrations of pro-inflammatory cytokines were reported to be neurotoxic ${ }^{26,27}$, which highlights the aggravating effects of unleashed PI-related (or even earlystage PI-related) inflammation on the neural system.

The irritation of nociceptors by pro-inflammatory cytokines causing 'inflammatory pain' as explained above, is one of the three main contributors to the overall pain sensation associated with exposure to sustained cell and tissue deformations and a resulting $\mathrm{PI}$ (or the early-stage, microscopic tissue damage that precedes a 'clinical' PI). Another main contributor to pathologic pain is the mechanical irritation applied by the increasing interstitial pressures on nociceptors as an inflammatory oedema builds up which causes direct 'nociceptive pain'. The third main contributor to pain is the ischaemic and acidotic biochemical conditions in the soft tissues at and near the site of a forming PI which would develop as the oedema compromises the blood perfusion, thereby inducing an 'ischaemic pain'. Accumulation of lactic acid in ischaemic tissue regions as hypoxic cells shift to an anaerobic metabolic pathway lowers the tissue $\mathrm{pH}$ which further stimulates nociceptors. Ischaemic tissues also exhibit a rise in extracellular adenosine triphosphate (ATP) levels, whereas extracellular ATP is also known to excite nociceptors and thereby contribute to deep tissue ischaemic pain ${ }^{28-31}$. The inextricable association between the vicious cycle of $\mathrm{PI}$ formation and the three main pathologic pain pathways - inflammatory pain, nociceptive pain and ischaemic pain, as described above - is depicted in Figure 2. This graphic description illustrates the parallel vicious cycle of multi-factorial pain that is strongly coupled to the main vicious cycle of the PI development.

Despite the fact that discomfort and pain sensations are always subjective, anecdotal clinical evidence collected by the authors demonstrates that alleviating tissue deformations increases the comfort of users who cannot respond to the above-described noxious stimuli (Figure 2). The testimony of Patient $A$ and the two case stories of Patients $B$ and $C$ (Figures 3-5) have been documented in interviews and clinical examinations of patients who used a powered, nonalternating, minimum tissue deformation (MTD) mattress with a double-cell structure and reactive adjustment technology which automatically maximises the patient-specific body envelopment at all times ${ }^{32}$.

Figure 3. Testimony of Patient A

Background and underlying conditions: I am 57 years old (male). My height is $175 \mathrm{~cm}$ and my bodyweight is approximately $80 \mathrm{~kg}$. I had a brain stroke in 1996. Diagnosis was "locked-in-syndrome" which indicates awareness but complete paralysis of all the voluntary muscles except from eye movements and blinking. I am unable to move and speak, but I can communicate with the help of a computer. My food must be mushed. My sense of touch exists. Maybe that has also helped, since I never had PIs or other skin problems.

Positioning and repositioning: In the evenings I go to bed at about 7pm or sometimes later. First I am lying on my back and watching television. Between 10pm and midnight I go to sleep. Nurses then turn me to a side position and once a night they turn me on my back. I spend about 11-12 hours in bed each night. During daytime, I am in bed for about 2 hours, when I am having my daily rest. When not in bed, I use an air-cell-based cushion on my wheelchair. I sit continuously for 4-5 hours; sometimes my daily rest fails and then I sit for 11-12 hours.

Comfort of use: My MTD mattress is adjusted with a handheld precision manometer (I can control pushbuttons with the help of my cheek). As I am in bed, the automatic controller will start to search the right pressure values. Thanks to the fact that I have my sense of touch, I adjust the mattress according to my own "feeling". At the moment the values of my MTD mattress are somewhere on the levels of $7 \mathrm{mmHg}$ at the shoulders, $11-12 \mathrm{mmHg}$ under the centre of the body and $5 \mathrm{mmHg}$ at the feet. I can say that $8 \mathrm{mmHg}$ in the middle of the mattress is too low for me, and $12 \mathrm{mmHg}$ feels better. I have used the MTD mattress for more than 20 years (since I was in rehabilitation) and never experienced pain. 
Patient B suffered from a riding accident in 2006, when she was 9 years old. Partial dislocation of vertebrae C1/2 in her cervical spine caused medullary damage which resulted in paralysis but with an ability to move her legs. Another result of that accident was a distension lesion of the brachial plexus on the left side of her body which caused a complex regional pain syndrome in her left upper extremity. Patient B suffered continuous headaches, pain in her neck and left upper extremity (at a level of 9-10 on a visual analogue scale and which did not resolve by transdermal pain relief patches), together with hyperalgesia. Accordingly, Patient B also had serious sleep problems. She was unable to sleep in a lateral position and usually slept half-seated and supported by multiple pillows.

When Patient B was 17 years old (and at a height of $150 \mathrm{~cm}$ and with a bodyweight of $58 \mathrm{~kg}$ at that time), her mother, a healthcare professional, had requested an MTD support surface for her daughter, based on a Braden score of 15 as well as the aforementioned half-seated sleeping position - both indicating a PI risk. After deploying the MTD mattress, Patient $\mathrm{B}$ and her mother reported that: "Previously falling asleep was very cumbersome and sleeping was fragmentary. Now the head of the bed could be lowered for supine sleeping, falling asleep is rapid with less pain and the sleep is continuous for up to 10 hours, after which the patient feels rested and refreshed. Daytime napping is also easy now whereas previously, this was possible only when the patient was extremely exhausted".

The "locked-in-syndrome" condition of Patient A (Figure 3) is relatively rare, as he is unable to move but is still able to sense. This rare condition provides a unique learning opportunity regarding the relations between sustained tissue deformations, discomfort and pain (Figure 2). Patient $A$ never developed Pls and his testimony is that, in addition, he has on no occasion experienced (pathologic) pain; this is consistent as he is sensate and if he had developed PI-related tissue damage that would have been painful (Figure 2). Importantly, the ability of Patient A to adjust his MTD mattress as he describes, by controlling the region-specific inflation pressures of the mattress each day, optimises the envelopment of his body contours head-to-toe, daily and for years, as the body changes throughout the years. This characteristic of the mattress is called 'adjustability"15,33 - the ability of the support surface to adjust to the changes in the body mass, tissue structure and composition that occur during life, for example due to growth and ageing. Such changes in the body structure and composition are often accelerated in patients who are at risk for Pls, typically due to the disuse atrophies in their tissues ${ }^{34}$. It is surmised that the fact that Patient A has never developed Pls, and did not experience pain either, is attributed to the adjustability of his MTD mattress.

Chronic inflammation of the CNS is a prominent and common condition in any state of extensive traumatic damage to the CNS, as well as in many childhood neurodegenerative diseases ${ }^{35}$. Chronic CNS inflammation was therefore very likely present in the cases of Patient B (CNS trauma; Figure 4) and Patient $C$ (CNS disease; Figure 5). Chronic inflammation of the CNS is also a main contributor to development of hyperalgesia ${ }^{25}$, as indeed was formally diagnosed in the case of Patient B (Figure 4). It is surmised that the arbitrary action of an alternating pressure system which is not able to account for the increased pain perception sensitivity of such hyperalgesic patients creates repetitive waves of rising tissue deformations (Figure 1) at and near the supported body regions. This might have stimulated the nociceptors with their reduced thresholds, causing them to fire with increased frequency in the inflammatory pain route described in Figure 2, which is a likely explanation to the severe restlessness of Patient $C$ when she was placed on an alternating pressure mattress (Figure 5). It is therefore postulated that patients who are unable to communicate but suffer CNS injuries or diseases - that typically involve chronic CNS inflammation leading to hyperalgesia - should not be placed on alternating pressure systems. Rather, such patients should use an MTD technology to alleviate the mechanical loading applied to their nociceptors, as opposed to cyclically ramping the loads as alternating pressure systems do. This approach has proven its effectiveness in the cases of both Patients B and C (Figures 4 and 5). With that said, the above point is still a hypothesis that requires research support by means of methodological studies.

In contrast to physiological signals such as the heart rate, blood pressure or body core temperature, pain is first and foremost a subjective experience and therefore cannot be measured in a strict sense. Noxious stimuli and nociceptive responses can be quantified from an electrophysiological (laboratory) perspective; however, the pain sensation itself is multi-faceted and includes mental, psychological, emotional, cognitive and social elements which are characteristic to the individual and specific to the time and circumstances ${ }^{36}$. Researchers often encounter these challenges by using semi-quantitative approaches including records of a visual analogue scale, verbal pain descriptors, numerical descriptor scales or scores of sleep quality and sleep patterns in order to report the pain outcomes. Nevertheless, the multi-faceted experience of patients and their individual circumstances are often lost through such 'data reduction' methodologies. Accordingly, here, the focus was on the patients and their care provider perspectives, which are largely missing in the literature, especially in an aetiological context. Additional work of both types, qualitative (patient case stories, desirably 
Figure 5. Case story of Patient C

At the age of 7 years Patient $\mathrm{C}$, a female, has been diagnosed with juvenile neuronal ceroid lipofuscinoses, a rare genetic progressive and degenerative neurometabolic disorder with fatal consequences. At the age of 30 years, Patient $\mathrm{C}$ (who was about $170 \mathrm{~cm}$ tall and weighed $85 \mathrm{~kg}$ at that time) was completely blind, bedridden and unable to speak or eat independently. In addition, Patient $\mathrm{C}$ suffered frequent epileptic seizures which did not respond to medications. The patient's mother had taken the responsibility of full-time care of her daughter, with occasional help from healthcare professionals. Patient $\mathrm{C}$ could express pain only as restlessness behaviour. Because of her high PI risk, an alternating mattress system has been utilised; however, the mother became worried since the epileptic seizures as well as the anguish episodes expressed by the restlessness doubled in frequency (based on the mother's rigorous daily logging of her daughter's condition and behaviour).

Carefully observing her daughter's restlessness behaviour patterns, the mother suspected that it was the alternating pressure cycles which were causing her daughter discomfort and pain but, for various reasons, the mother was unable to change the support surface of her daughter for 8 months. After that period, an opportunity formed to replace the alternating pressure system by a non-alternating MTD support surface. Immediately after that change was made, Patient $\mathrm{C}$ became more calm and peaceful during both day and night times and, therefore, the pain medication dose could be reduced to half. Interestingly, during the last 6 weeks on the alternating pressure system, the patient had 12 epileptic seizures compared to only five seizures over the first 6 -week period on the MTD mattress.

Another major change documented by the mother was that her daughter could sleep continuously for 10 hours at night and, in addition, often take daytime naps, whereas on the alternating pressure system her night sleep was always uneasy and fragmentary and daytime napping was rare. The mother described a substantial improvement of her daughter's quality of life starting when the MTD mattress has been put into use.

Patient C passed away on October 2012 at the age of 31 years.

told by the patients themselves) and semi-quantitative studies (using the aforementioned measures) is warranted in order to shed more light on this fundamentally important consequence of being at risk for a $\mathrm{PI}$ or suffering a $\mathrm{PI}$. Likewise, additional research with regards to the roles of different support surfaces in this relationship between pain and Pls is needed, again employing both qualitative and semi-quantitative approaches.

With reference to the more up-to-date studies published in the last 2 decades, surprisingly little published information is available to correlate different support surface technologies with semi-quantitative pain measures. Despite the fact that researchers have mentioned anecdotally that the type of the support surface is associated with improvement in the reported discomfort or pain levels ${ }^{37,38}$, there are no head-tohead support surface comparisons focusing on discomfort and pain which have assessed the discomfort or pain levels in the same individuals after they have experienced two or more different support surface types. Studies comparing (dis) comfort levels in relatively small groups ${ }^{39}$ are limited in this regard, as the aforementioned individualised multi-factorial subjective elements, including potential learned behaviours to cope with the discomfort or pain, are not accounted for in these cohort comparisons. Though documented qualitatively, the case reports presented here, in particular for Patients $B$ and $\mathrm{C}$, are unique in this regard.

\section{Conclusion}

This article provided an overview of the contemporary research findings and latest aetiological discoveries concerning the pathophysiology of Pls as reported in the 2019 International guideline ${ }^{5}$, with some relevant updates in relation to the COVID-19 pandemic. The fundamental biomechanical principles of PIP with regards to the key characteristics of support surfaces were also explained here. In addition, the relations between alleviation of sustained cell and tissue deformations, discomfort and pain were also reviewed here as a new update to the information in the 2019 International guideline ${ }^{5}$.

The aetiological links between the main vicious cycle of PI formation which is triggered and driven by exposure to sustained cell and tissue deformations, and the coupled vicious cycle of pain, which is fed by the inflammation, oedema and ischaemia, are important to consider in clinical practice. The latter point was addressed through a discussion of the current PI aetiology theory in the context of one documented patient testimony and two additional patient case stories which have been used to analyse the complex interlinks between the known aetiological factors in Pls - discomfort and pain. In addition, the alleviation of sustained cell and tissue deformations protects from Pls and is pivotal in successful PIP; reduced exposure to tissue deformations may decrease the discomfort and pain that may be associated with early-stage, evolving or existing Pls.

\section{Conflict of interest}

The authors are consultants to Carital Ltd (Helsinki, Finland) whose MTD mattress technology is referred to in this paper. These relationships had no influence on the literature review nor interpretation of the clinical data reported here. 


\section{Funding}

The authors received no funding for this study.

\section{References}

1. Gefen A, Alves P, Ciprandi G, Coyer F, Milne CT, Ousey K, Ohura N, Waters N, Worsley P. Device-related pressure ulcers: SECURE prevention. J Wound Care 2020;29(Sup2a):S1-S52.

2. Gefen A, Ousey K. Update to device-related pressure ulcers: SECURE prevention. COVID-19, face masks and skin damage. J Wound Care 2020;29(5):245-259.

3. Gefen A. The future of pressure ulcer prevention is here: detecting and targeting inflammation early. EWMA J 2018;19(2):7-13.

4. Gefen A. How medical engineering has changed our understanding of chronic wounds and future prospects. Med Eng Phys 2019;72:13-8.

5. Gefen A, Brienza D, Edsberg L, Milton W, Murphy C, Oomens CWJ, Perry L, Sari Y. The etiology of pressure injuries. In: European Pressure Ulcer Advisory Panel (EPUAP), National Pressure Injury Advisory Panel (NPIAP), Pan Pacific Pressure Injury Alliance (PPPIA). Prevention and treatment of pressure ulcers/injuries: clinical practice guidelines. The international guideline. 3rd ed. Emily Haesler, editor. EPUAP, NPIAP, PPPIA; 2019.

6. Gefen A. How much time does it take to get a pressure ulcer? Integrated evidence from human, animal, and in vitro studies. Ostomy Wound Manage 2008;54(10):26-35.

7. Gefen A, Weihs D. Cytoskeleton and plasma-membrane damage resulting from exposure to sustained deformations: a review of the mechanobiology of chronic wounds. Med Eng Phys 2016;38(9):828-33.

8. Soetens JFJ, Worsley PR, Bader DL, Oomens CWJ. Investigating the influence of intermittent and continuous mechanical loading on skin through non-invasive sampling of IL-1 $1 \alpha$. J Tissue Viability 2019;28(1):1-6.

9. Traa WA, van Turnhout MC, Nelissen JL, Strijkers GJ, Bader DL, Oomens CWJ. There is an individual tolerance to mechanical loading in compression induced deep tissue injury. Clin Biomech 2019;63:153-60.

10. Schett G, Sticherling M, Neurath MF. COVID-19: risk for cytokine targeting in chronic inflammatory diseases? Nat Rev Immunol 2020;20:271-72.

11. Long B, Brady WJ, Koyfman A, Gottlieb M. Cardiovascular complications in COVID-19. Am J Emerg Med 2020; in press (available online), doi:10.1016/j.ajem.2020.04.048

12. Spiezia L, Boscolo A, Poletto F, Cerruti L, Tiberio I, Campello E, Navalesi P, Simioni P. COVID-19-related severe hypercoagulability in patients admitted to intensive care unit for acute respiratory failure. Thromb Haemost 2020;120(6):998-1000.

13. Phua J, Weng L, Ling $L$, et al. Intensive care management of coronavirus disease 2019 (COVID-19): challenges and recommendations. Lancet Respir Med 2020;8(5):506-17.

14. Ahtiala M, Kivimäki R, Laitio R, Soppi E. The association between pressure ulcers/injuries development and short-term mortality in critically ill patients: a retrospective cohort study. Wound Manage Prevent 2020;66(2):14-21.

15. Levy A, Shoham N, Kopplin K, Gefen A. The critical characteristics of a good wheelchair cushion. In: Romanelli M, Clark M, Gefen A, Ciprandi G, editors. Science and practice of pressure ulcer management, 2nd Ed. ISBN: 978-1-4471-7411-0. London: Springer-Verlag; 2018 p. 17-31.

16. Call E, Cheney A. Developing standard test methods for assessment of medical devices in the field of wound prevention and care. In: Gefen, A. Innovations and emerging technologies in wound care. London: Academic Press (Elsevier); 2020.

17. Schwartz D, Magen YK, Levy A, Gefen A. Effects of humidity on skin friction against medical textiles as related to prevention of pressure injuries. Int Wound J 2018;15(6):866-74.
18. Zeevi T, Levy A, Brauner N, Gefen A. Effects of ambient conditions on the risk of pressure injuries in bedridden patients: multi-physics modelling of microclimate. Int Wound J 2018;15(3):402-16.

19. Gouin JP, Kiecolt-Glaser JK. The impact of psychological stress on wound healing: methods and mechanisms. Immunol Allergy Clin Noth Am 2011;31(1):81-93.

20. Gold MS, Gebhart GF. Nociceptor sensitization in pain pathogenesis. Nat Med 2010;16(11):1248-57.

21. Apsingi S, Dussa CU. Can peripheral nerve blocks contribute to heel ulcers following total knee replacement? Acta Orthop Belg 2004 Oct;70(5):502-4.

22. Kulmatycki KM, Jamali F. Drug disease interactions: role of inflammatory mediators in pain and variability in analgesic drug response. J Pharm Pharm Sci 2007;10(4):554-66.

23. Hess A, Axmann R, Rech J, Finzel S, Heindl C, Kreitz S, Sergeeva M, Saake M, Garcia M, Kollias G, Straub RH, Sporns O, Doerfler A, Brune K, Schett $G$. Blockade of TNF- $\alpha$ rapidly inhibits pain responses in the central nervous system. Proc Natl Acad Sci USA 2011;108(9):3731-6.

24. Zhou YQ, Liu Z, Liu ZH, Chen SP, Li M, Shahverano A, Ye DW, Tian YK. Interleukin-6: an emerging regulator of pathological pain. J Neuroinflammation 2016;13(1):141.

25. Reinold H, Ahmadi S, Depner UB, Layh B, Heindl C, Hamza M, Pahl A, Brune K, Narumiya S, Müller U, Zeilhofer HU. Spinal inflammatory hyperalgesia is mediated by prostaglandin $\mathrm{E}$ receptors of the EP2 subtype. J Clin Invest 2005;115(3):673-9.

26. Czirr E, Wyss-Coray T. The immunology of neurodegeneration. J Clin Invest 2012;122(4):1156-63.

27. Brambilla R. Neuroinflammation, the thread connecting neurological disease: cluster: "Neuroinflammatory mechanisms in neurodegenerative disorders". Acta Neuropathol 2019;137(5):689691.

28. Inoue $\mathrm{K}$, Tsuda M, Koizumi S. ATP receptors in pain sensation: involvement of spinal microglia and P2X(4) receptors. Purinergic Signal 2005;1(2):95-100.

29. Melani A, Turchi D, Vannucchi MG, Cipriani S, Gianfriddo M, Pedata F. ATP extracellular concentrations are increased in the rat striatum during in vivo ischemia. Neurochem Int 2005;47(6):442-8.

30. Birdsong WT, Fierro L, Williams FG, Spelta V, Naves LA, Knowles M, Marsh-Haffner J, Adelman JP, Almers W, Elde RP, McCleskey EW. Sensing muscle ischemia: coincident detection of acid and ATP via interplay of two ion channels. Neuron 2010;68(4):739-49.

31. Anitescu M. Ischemic pain. In: Cheng J, Rosenquist R, editors. Fundamentals of pain medicine. Springer; 2018, p.141-51.

32. Ahtiala M, Kivimäki R, Laitio R, Soppi E. Effect of mattress deployment on pressure ulcer development: a real-world observational cohort experience. Wounds Int 2020;11(1):10-7.

33. Levy A, Kopplin K, Gefen A. Adjustability and adaptability are critical characteristics of pediatric support surfaces. Adv Wound Care (New Rochelle) 2015;4(10):615-22.

34. Gefen A. Tissue changes in patients following spinal cord injury and implications for wheelchair cushions and tissue loading: a literature review. Ostomy Wound Manage 2014;60(2):34-45.

35. Lim M. Treating inflammation in childhood neurodegenerative disorders. Dev Med Child Neurol 2011;53(4):298-304.

36. Upton D, Solowiej K. Pain and stress as contributors to delayed wound healing. Wound Practice Res 2010;18:114-22.

37. Girouard K, Harrison MB, Van Den Kerkof E. The symptom of pain with pressure ulcers: a review of the literature. Ostomy Wound Manage 2008;54(5):30-42.

38. Gleeson D. Effectiveness of a pressure-relieving mattress in an acute stroke ward. Br J Nurs 2016;25(20 Suppl):S34-S38.

39. Vermette S, Reeves I, Lemaire J. Cost effectiveness of an airinflated static overlay for pressure ulcer prevention: a randomized, controlled trial. Wounds 2012;24(8):207-14. 